\title{
Equoterapia como proposta de intervenção para pacientes com Paralisia Cerebral: revisão integrativa
}

\author{
Equotherapy as a proposed intervention for patients with Cerebral Paralysis: integrative \\ review
}
Equoterapia como propuesta de intervención para pacientes con Paralisia Cerebral: revisión integrativa

Fernando Ribeiro Castro ${ }^{1 *}$; Marcio Marinho Magalhães².

\begin{abstract}
RESUMO
Objetivo: Investigar na literatura cientifica os efeitos da equoterapia em pacientes com paralisia cerebral. Métodos: Trata-se de uma revisão integrativa, de estudos disponíveis nas bases de dados Scielo e Biblioteca Virtual em Saúde, publicados entre os anos de 2010 a 2017 nos idiomas português e inglês, no qual totalizaram 9 estudos. Resultados: Pode-se verificar em várias pesquisas que a equoterapia foi capaz de melhorar a capacidade funcional, a função motora grossa, a autoestima, capacidade de autocuidado, comunicação e cognição social. O cavalo é capaz de gerar e transmitir ao paciente vários movimentos de maneira sequenciada e simultânea, nesse sentido a equoterapia em pacientes com paralisia cerebral é capaz de proporcionar o controle postural, através do estímulo das reações normais e por meio de estímulos repetitivos de coordenação da postura. Conclusão: Portanto pode-se verificar que a equoterapia possui benefícios no tratamento de alterações funcionais e até mesmo de caráter social e emocionais em pacientes com paralisia cerebral.
\end{abstract}

Palavras-chave: Equoterapia, Paralisia Cerebral, Fisioterapia.

\begin{abstract}
Objective: To investigate in the scientific literature the effects of equine therapy in patients with cerebral palsy. Methods: This is an integrative review of studies available in the Scielo and Virtual Health Library databases, published between the years 2010 and 2017 in the Portuguese and English languages, in which there were 9 studies. Results: It can be verified in several researches that equine therapy was able to improve functional capacity, gross motor function, self-esteem, capacity for self-care, communication, social cognition. The horse is able to generate and transmit to the patient several movements in a sequenced and simultaneous way, in this sense the equotherapy in patients with cerebral palsy is able to provide the postural control, through the stimulation of the normal reactions and through repetitive stimuli of coordination of the posture. Conclusion: Therefore, it can be verified that equotherapy has benefits in the treatment of functional and even social and emotional changes in patients with cerebral palsy.
\end{abstract}

Key words: Equine Therapy, Cerebral Palsy, Physiotherapy.

\section{RESUMEN}

Objetivo: Investigar en la literatura científica los efectos de la equinoterapia en pacientes con parálisis cerebral. Métodos: Se trata de una revisión integradora de los estudios disponibles en bases de datos SciELO y Biblioteca Virtual en Salud, publicados entre 2010 a 2017 en portugués y en Inglés, que ascendió a nueve estudios. Resultados: Se puede verificar en varias investigaciones que la ecuoterapia fue capaz de mejorar la capacidad funcional, la función motora gruesa, la autoestima, capacidad de autocuidado, comunicación,

\footnotetext{
${ }^{1}$ Fisioterapeuta Graduado pela Faculdade Santa Terezinha (CEST), São Luis (MA).

*E-mail: fisnando@yahoo.com.br

${ }^{2}$ Fisioterapeuta Graduado pela Faculdade Centro Universitário de Ciências e Tecnologia do Maranhão (UNIFACEMA), Caxias (MA).
}

SUBMETIDO EM: 1/2019 
cognición social. El caballo es capaz de generar y transmitir al paciente varios movimientos de manera secuenciada y simultánea, en ese sentido la equinoterapia en pacientes con parálisis cerebral es capaz de proporcionar el control postural, a través del estímulo de las reacciones normales y por medio de estímulos repetitivos de coordinación de la enfermedad postura. Conclusión: Por lo tanto, se puede verificar que la equinoterapia tiene beneficios en el tratamiento de alteraciones funcionales e incluso de carácter social y emocionales en pacientes con parálisis cerebral.

Palabras clave: Hipoterapia, Parálisis cerebral, Fisioterapia.

\section{INTRODUÇÃO}

A paralisia cerebral consiste em um conjunto de patologias que afetam o desenvolvimento da postura e dos movimentos, que podem promover a incapacidade de realização de atividades motoras, ocasionado por alterações no cérebro durante a fase de crescimento fetal ou infantil (COVER et al., 2017).

Segundo Dias et al. (2010); crianças com paralisia cerebral apresentam principalmente alterações no sistema motor, com ênfase na biomecânica corporal, sendo que podem ainda apresentar modificações cognitivas, sensitivas, na visão, auditivas, gerando Danos para o desempenho funcional dessas crianças repercutindo de forma significa tica na realização de tarefas diárias.

Preconiza-se para o tratamento da paralisia cerebral a utilização de medicamentos e quando necessária intervenção cirúrgica, entre os fármacos indicados destaca-se os anticonvulsivantes e antiespásticos. Em relação aos procedimentos cirúrgicos englobam cirurgias ortopédicas para corrigir alterações articulares, com intuito de preservar a função e avaliar a dor (COVER et al., 2017).

De acordo com Copetti et al. (2007); existem vários métodos de tratamento da paralisia cerebral, dentre os quais destaca-se a equoterapia como um recurso terapêutico e educacional, em que o cavalo é um instrumento interdisciplinar e que promove o desenvolvimento biopsicossocial.

A equoterapia é indicado por médico e existe a necessidade de uma avaliação prévia do paciente, baseiase em fundamentos científicos, no qual usa o cavalo em um contexto multiprofissional e interdisciplinar. Esse tratamento é feito com auxílio de profissionais de várias áreas como saúde, educação e equitação, que auxiliam a reabilitar pessoas com necessidades especiais, não somente em relação aos movimentos, mas também a melhorar autoestima e autoconfiança (ARAUJO et al., 2010).

Segundo Becheva et al. (2016); a equoterapia trata-se de uma intervenção fisioterapêutica que colabora para melhora na funcionalidade geral e independência para realização das atividades de vida diária, sendo que alguns autores já sugerem estes benefícios para indivíduos com diagnóstico de paralisia cerebral. Portanto este trabalho teve como objetivo principal investigar na literatura cientifica os efeitos da equoterapia em pacientes com paralisia cerebral.

Portanto este trabalho teve como objetivo principal investigar na literatura cientifica os efeitos da equoterapia em pacientes com paralisia cerebral.

\section{METODOLOGIA}

Este estudo utiliza como método a revisão integrativa, a qual tem como finalidade reunir e resumir o conhecimento científico já produzido sobre o tema "Equoterapia como recurso terapêutico em crianças com paralisia cerebral" para permitir buscar, avaliar e sintetizar as evidências disponíveis para contribuir com o desenvolvimento do conhecimento na temática. Foram incluídos trabalhos disponíveis nas seguintes bases de dados: "Scielo", "Mdeline" e "Lilacs" Critérios de inclusão: Estudos publicados no período de 2007 a 2017 , nos idiomas português e inglês. Foram excluídos resumos, artigos com equívocos metodológicos, teses, dissertações, artigos de revisão e que não contivessem ano de publicação, volume e número em revista. Os descritores utilizados foram: "Equoterapia", "Paralisia Cerebral"; "Fisioterapia". Após as buscas nas bases de dados utilizando a associação (Equoterapia AND Paralisia Cerebral AND Fisioterapia), foram encontrados 588 artigos, mediante aplicação dos critérios de inclusão ficaram 278 estudos publicados. Destes, após a 
aplicação dos critérios de exclusão, restaram 58 publicações. Dessas, 09 publicações apresentavam metodologia, temática e objetivos condizentes com os objetivos da pesquisa atual (Figura 1).

Figura 1 - Representação gráfica da aplicação dos critérios de inclusão e exclusão.

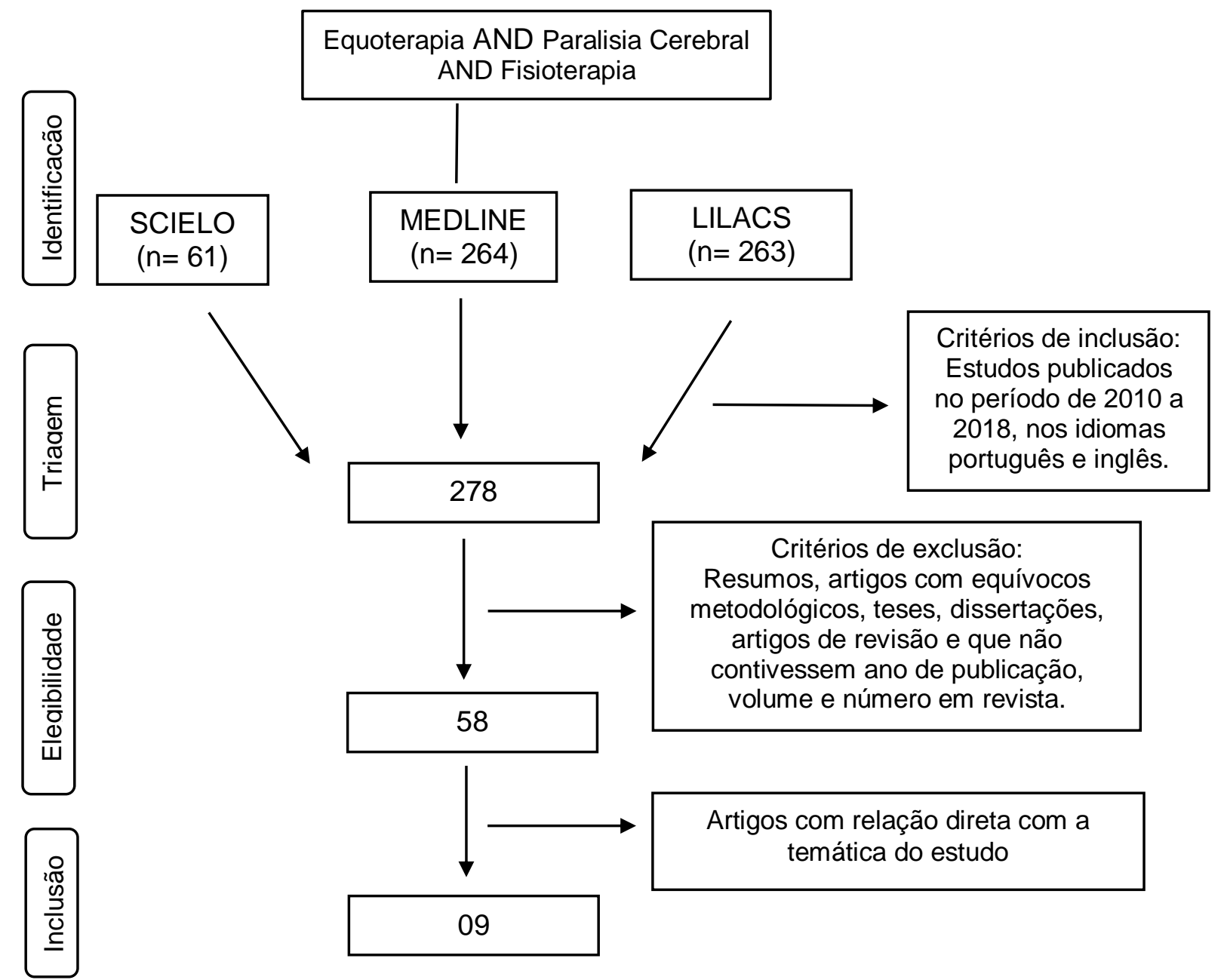

Fonte: Pesquisa direta, 2019.

A tabela 1 evidencia a distribuição os estudos de acordo com o ano de publicação. $O$ ano de 2017 apresentou o maior número de publicações correspondendo a 44,5\% do total. Nos anos de 2016 e 2013 foram identificadas 1 publicação em cada, correspondendo a 11,1\% cada. Por fim no ano de 2011 foram publicados 3 estudos, representando $33,3 \%$, respectivamente.

Tabela 1 - Distribuição dos estudos incluídos, segundo o ano de publicação.

\begin{tabular}{ccc}
\hline Ano da publicação & Número absoluto & $\%$ \\
\hline 2017 & 4 & 44,5 \\
2016 & 1 & 11,1 \\
2013 & 1 & 11,1 \\
2010 & 3 & 33,3 \\
TOTAL & 9 & $100 \%$ \\
\hline
\end{tabular}

Fonte: Pesquisa direta, 2019. 
A tabela 2 mostras a distribuição dos estudos por idioma. Houve uma prevalência é 84,4\% de publicações no idioma inglês. E somente $15,4 \%$ para o idioma português.

Tabela 2 - Distribuição dos artigos de acordo com o idioma.

\begin{tabular}{lcc}
\hline Idioma & Número absoluto & $\%$ \\
\hline Português & 6 & 66,7 \\
Inglês & 3 & 33,3 \\
\hline TOTAL & 9 & $100 \%$
\end{tabular}

Fonte: Pesquisa direta, 2019.

\section{RESULTADOS E DISCUSSÃO}

Na Tabela 1 apresenta a distribuição das publicações quanto ao ano, autores, e principais resultados relacionados as contribuições da Equoterapia como proposta de intervenção para pacientes com Paralisia Cerebral. As publicações estão dispostas em ordem cronologia e alfabética, a partir da publicação mais atual.

Tabela 1 - Publicações relacionados as contribuições da Equoterapia.

\begin{tabular}{|c|c|c|c|}
\hline Trabalhos & Ano & Autor(es) & Resultados \\
\hline T1 & 2017 & CORRÊA RG, et al. & $\begin{array}{l}\text { Houve melhora na capacidade de } \\
\text { permanecer em pé, no alinhamento postural, } \\
\text { porém não houve um score significativo dos } \\
\text { resultados obtidos }\end{array}$ \\
\hline T2 & 2017 & FERREIRA, et al. & $\begin{array}{l}\text { As crianças apresentaram aumento na } \\
\text { pontuação total da MIF. Houve melhora nos } \\
\text { itens relativos à autocuidados, mobilidade, } \\
\text { locomoção, comunicação e cognição social }\end{array}$ \\
\hline T3 & 2017 & PIETRO AV. & $\begin{array}{l}\text { Promoveu benefícios na função motora } \\
\text { grossa e desempenho funcional, melhora nos } \\
\text { parâmetros de autocuidado e mobilidade }\end{array}$ \\
\hline T4 & 2017 & VIDAL LR, et al. & $\begin{array}{l}\text { O praticante foi apresentando autonomia ao } \\
\text { montar no cavalo e permanecer nele com } \\
\text { mais segurança, também a melhora da } \\
\text { autoestima }\end{array}$ \\
\hline T5 & 2016 & ROSAN L, et al. & $\begin{array}{l}\text { Melhora expressiva da qualidade de vida e } \\
\text { participação das crianças com paralisia } \\
\text { cerebral nos aspectos relacionados ao } \\
\text { envolvimento no contexto social }\end{array}$ \\
\hline T6 & 2013 & GREGÓRIO A, KRUEGER E. & $\begin{array}{l}\text { A equoterapia apresentou grande eficiência } \\
\text { no ganho do controle cervical e de tronco }\end{array}$ \\
\hline T7 & 2010 & ARAUJO AERA, et al. & $\begin{array}{l}\text { Os menores benefícios posturais foram } \\
\text { observados nos segmentos cabeça e } \\
\text { pescoço }(73 \%) \text { e ombros e escápula ( } 84 \%) \text {, } \\
\text { enquanto que os maiores foram no tronco } \\
(93 \%) \text { e na pélvis }(114 \%) \text {. }\end{array}$ \\
\hline T8 & 2010 & GALVÃO A, et al. & $\begin{array}{l}\text { A equoterapia proporcionou melhora do } \\
\text { equilíbrio, postura e coordenação. }\end{array}$ \\
\hline T9 & 2010 & NASCIMENTO MVM, et al. & $\begin{array}{l}\text { Observou-se que ao realizar } 30 \text { sessões de } \\
\text { equoterapia com duração de } 30 \text { minutos } \\
\text { tiveram melhora significativa das crianças em } \\
\text { relação a capacidade de sentar }\end{array}$ \\
\hline
\end{tabular}

Fonte: Pesquisa direta, 2019. 
No estudo de caso desenvolvido por Correâ RG et al. (2017), com uma criança com paralisia cerebral atetóide do sexo feminino, com 5 anos de idade, no qual realizou-se dez seções de equoterapia e avaliou-se com a Escala de Equilíbrio de Berg, antes e após o tratamento, verificou-se que houve melhora em relação a capacidade de passar da posição em pé para posição sentada e permanecer em pé sem apoio com os pés junto.

No trabalho "Análise qualitativa do efeito da equoterapia para crianças com paralisia cerebral", os autores observaram que os três pacientes avaliados apresentaram aumento da pontuação total da MIF (Medida de Independência Funcional), destaca-se melhora para realização das atividades de vida diárias como autocuidados, mobilidade, locomoção, comunicação e cognição social (FERREIRA et al., 2017).

Uma pesquisa que teve como objetivo analisar se a frequência semanal das sessões de equoterapia influenciaria na função motora grossa e no desempenho funcional em crianças com paralisia cerebral, averiguou-se que em relação a funcionalidade houve diferença estatisticamente significativa no autocuidado e mobilidade, mas em relação a função motora grossa não obteve diferença estatisticamente significativa (PIETRO AV, 2017).

No estudo de Rosan L, et al. (2016), em que todos os participantes apresentavam comprometimento motor severo, os autores observaram um aumento dos escores após a intervenção com a equoterapia, no qual destaca-se o impacto na qualidade de vida dos participantes.

Enquanto Gregório A e Krueger E. (2013), avaliaram a influência da equoterapia no controle cervical e de tronco em uma criança com paralisia cerebral, observou-se melhora em relação a simetria corporal, como controle cervical e torácico, assim como aumento da motricidade dos membros inferiores e superiores.

A literatura cientifica aponta que a equoterapia em pacientes com paralisia cerebral é capaz de proporcionar o controle postural, através do estímulo das reações normais e por meio de estímulos repetitivos de coordenação da postura (ESPINDULA AP et al., 2012).

Outra pesquisa com paciente com paralisia cerebral no qual foi participou de seções de equoterapia, observou-se melhora no equilíbrio avaliado através da Escala de Equilíbrio de Berg, notou-se também melhor alinhamento da postura (GALVÃO A et al., 2010).

A reabilitação de pessoas com necessidades especiais com limitações motoras, deficiência física, mental e psicológica pode ser obtida por meio da equoterapia, pois o cavalo é usado como um agente facilitador, porque proporciona aos pacientes adequação do tônus muscular, melhora na coordenação e no equilíbrio corporal (JANG CH et al., 2016).

Segundo Hsieh YL et al. (2016), a equoterapia é uma opção que colabora para a melhoria da qualidade de vida de pessoas com paralisia cerebral, principalmente as que não caminham ou que passam maior parte do tempo sentadas. O cavalo é capaz de gerar e transmitir ao paciente vários movimentos de maneira sequenciada e simultânea. No plano horizontal gera-se movimentos da direita para esquerda; no plano transversal gera-se movimentos para frente e para trás, cabe ressaltar que esta sequência de movimentos é amplamente complexa (NASCIMENTO MVM et al., 2010).

É relevante destacar que o tratamento para paralisia cerebral necessita de uma equipe multiprofissional, para tratar as alterações de postura, deformidades e assimetrias, em vista que o intuito principal dos tratamentos para esta patologia é melhorar a capacidade funcional (ARAUJO AERA, RIBEIRO VS, SILVA BTF, 2010).

\section{CONCLUSÃO}

Portanto pode-se verificar que a equoterapia possui benefícios no tratamento de alterações funcionais e até mesmo de caráter social e emocionais em pacientes com paralisia cerebral, em vista que os estudos analisados demostraram efeitos positivos. Contudo encontrou-se algumas limitações, como poucos estudos com uma amostra significativa pois a maioria dos trabalhos eram estudos de casos. 


\section{REFERÊNCIAS}

1. COVER et al. Cerebral palsy. Lancet [internet]. 2017; 383(9924): 1240-1249.

2. DIAS et al. Desempenho funcional de crianças com paralisia cerebral participantes de tratamento multidisciplinar. Fisioterapia e Pesquisa [internet]. 2010;17(3): 225-229.

3. COPETTI F et al. Comportamento angular do andar de crianças com Síndrome de Down após intervenção com equoterapia. Rev Bras Fisioterapia [internet]. 2007; 11(6): 503-507.

4. BECHEVA M et al. integrated approach in children with cerebral palsy (CP). World Journal of Pharmacy and Pharmaceutical Sciences. [internet]. 2016; 5(7): 9-17.

5. CORRÊA RG, TONON E, SUTER TMC. A influência da equoterapia no equilíbrio de pacientes com paralisia cerebral. Revista Horus [internet]. 2017; 7(3): 1-8.

6. FERREIRA et al. Análise qualitativa do efeito da equoterapia para crianças com paralisia cerebral. Cadernos de Pósgraduação em Distúrbios do Desenvolvimento [internet]. 2017; 17(1): 62-68.

7. PIETRO AV. Efeitos da frequência semanal de um programa de equoterapia na função motora grossa e no desempenho funcional em crianças com paralisia cerebral. Dissertação (Mestrado em Educação Física) - Faculdade de Educação Física. Universidade de Brasília, Brasília, 2017, 117p.

8. VIDAL LR, CUNHA A, COSTA LPD. O desenvolvimento perceptivo de uma criança com paralisia cerebral através do contato com o cavalo: a importância do ambiente lúdico e seus aspectos positivos. Revista Interdisplinar de Ensino, Pesquisa e Extensão [internet]. 2017; 5(1): 29-44.

9. ROSAN L, BRACCIALLI LMP, ARAUJO RCT. Contribuição da equoterapia para a participação e qualidade de vida do praticante com paralisia cerebral em diferentes contextos. Revista Diálogos e Perspectivas em Educação Especial [internet]. 2016; 3 (1): 48-61.

10. GREGÓRIO A, KRUEGER E. A influência da equoterapia no controle cervical e de tronco em uma criança com paralisia cerebral. Revista UNIANDRADE 2013; 14(1): 65-75 65.

11. ARAUJO AERA, RIBEIRO VS, SILVA BTF. A equoterapia no tratamento de crianças com paralisia cerebral no Nordeste do Brasil. Revista Fisioterapia Brasil [Internet]. 2010; 11(1): 4-8.

12. GALVÃO A et al. Estudo de Caso: a equoterapia no tratamento de um paciente adulto portador de ataxia cerebelar. Rev Neurocienc 2010;18(3):353-358.

13. NASCIMENTO MVM et al. O valor da equoterapia voltada para o tratamento crianças com paralisia cerebral quadriplégica. Brazilian Journal of Biomotricity [internet]. 2010; 4(1): 48-56.

14. FERLINI GMS, CAVALARI N. Os benefícios da equoterapia no desenvolvimento da criança com deficiência física. Caderno Multidisciplinar de Pós-graduação [internet]. 2010; 1(4): 1-14.

15. ESPINDULA AP et al. Flexibilidade muscular em indivíduos com deficiência intelectual submetidos à equoterapia: estudo de casos. Rev Cienc Ext [internet]. 2012; 8(2): 125-133.

16. JANG CH et al. Effects of hippotherapy on psychosocial aspects in children with cerebral palsy and their caregivers: a pilot study. Annals of rehabilitation medicine [internet]. 2016; 40(2): 203-236.

17. HSIEH YL et al. Effects of hippotherapy on body functions, activities and participation in children with cerebral palsy based on ICF-CY assessments. Disability and Rehabilitation [internet]. 2016; 39(17): 1703-1713. 